\title{
Estudio de la insolvencia empresarial en las cooperativas mediante técnicas multivariantes ${ }^{*}$
}

\author{
JOSÉ POZUELO CAMPILLO \\ Departamento de Contabilidad, UNIVERSIDAD DE VALENCIA, ESPAÑA. E-mail: \\ Jose.Pozuelo@uv.es
}

\section{JULIÁN MARTÍNEZ VARGAS}

Departamento de Contabilidad, UNIVERSIDAD DE VALENCIA, ESPAÑA. E-mail: Julian.martinez@uv.es

\section{PEDRO CARMONA IBÁÑEZ}

Departamento de Contabilidad, UNIVERSIDAD DE VALENCIA, ESPAÑA. E-mail: Pedro.carmona@uv.es

\section{RESUMEN}

Una de las consecuencias de la actual crisis económica está siendo un significativo repunte de fracasos empresariales, lo que está avivando el interés de investigadores y usuarios por su análisis y estudio y la actualización de los modelos tradicionales.

Al revisar la literatura financiera sobre el fracaso empresarial en nuestro país, advertimos que son muy pocos los trabajos centrados exclusivamente en las empresas cooperativas, a pesar de ser muy numerosa esta fórmula empresarial en nuestro tejido productivo. Esta ausencia tan notoria de trabajos sobre este tipo de empresas, la disposición en la actualidad de grandes y completas bases de datos y las circunstancias económicas actuales nos han motivado para acometer este trabajo cuyo principal objetivo se centra en la estimación, a partir de técnicas estadísticas, de modelos aptos para la predicción del fracaso empresarial en las empresas cooperativas.

Palabras clave: Economía social, cooperativas, fracaso empresarial, Logit.

\section{Corporate Insolvency Studies in the Cooperative by Multivariate Techniques}

\begin{abstract}
One of the consequences of the current economic crisis is a significant rise in business failures, which is fueling the interest of researchers and users for its analysis, review and update of the traditional models.

In reviewing the financial literature on business failure in our country, we noticed that the are very few studies focusing exclusively on cooperative enterprises, although this kind of companies have a broad presence in our country. This significant lack of studies on this kind of business, the recent availability of large databases and the current economic circumstances have influenced us to undertake this work. The main paper objective focuses on the estimation of appropriate models to predict business failure in cooperative enterprises, using statistical techniques.
\end{abstract}

Keywords: Social Economy, Cooperatives, Business Failure, Logit.

Clasificación JEL: P1

\footnotetext{
* Trabajo realizado gracias a la financiación conseguida de la Generalitat Valenciana para la realización de proyectos de I+D para grupos de investigación emergentes mediante el proyecto GV/2012/052 correspondiente al ejercicio 2012.
}

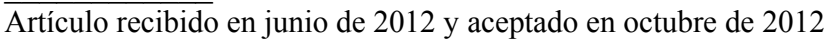

Artículo disponible en versión electrónica en la página www.revista-eea.net, ref. ə-30306 


\section{INTRODUCCIÓN}

El presente contexto de crisis económica y financiera y su dilatación en el tiempo está suponiendo la creciente desaparición de numerosas empresas, lo que ha provocado una revitalización del estudio de las causas que sumergen a las empresas en situaciones de dificultad financiera que en algunos casos pueden llegar a suponer su definitiva desaparición.

El volumen de investigaciones orientadas a la elaboración de modelos cuantitativos y cualitativos que permitan anticipar las situaciones de fracaso empresarial ha dependido frecuentemente de la etapa del ciclo económico que se estaba viviendo. Han sido frecuentes en los años de crisis y no lo han sido tanto en etapas de coyuntura alcista. Sin embargo, cuando se realiza una revisión de la literatura, fundamentalmente en la ultima década, se observa que son muy escasos los trabajos centrados en las pequeñas empresas, a pesar de conformar la mayor parte del tejido empresarial, y prácticamente inexistentes los que se han centrado en las cooperativas. Tal vez la ausencia de bases de datos fiables que impedían alcanzar un tamaño mínimo en las muestras de estudio para que los resultados fuesen estadísticamente significativos o la normativa contable singular que regula estas empresas ha impedido la consolidación de una línea de investigación específica en este sector empresarial ${ }^{1}$.

En este trabajo nos proponemos como principal objetivo desarrollar modelos cuantitativos que permitan describir y anticipar la probabilidad de que una empresa cooperativa se vea envuelta en procedimientos concursales a partir del estudio y tratamiento de la información económico-financiera suministrada por la propia firma.

Para alcanzar este fin llevaremos a cabo diversos análisis estadísticos sobre unas muestras de datos financieros de cooperativas españolas. Intentaremos, asimismo, evaluar si la información contable contenida en sus estados financieros es útil con fines descriptivos y predictivos, estudiando si es acertado su empleo sin cuestionarse su fiabilidad.

\section{ESPECIFICIDADES DE LAS EMPRESAS COOPERATIVAS}

Las cooperativas son sociedades de marcado carácter social cuyo objetivo es facilitar a sus socios determinados bienes o servicios al mejor precio (cooperativas de consumo) o retribuir sus prestaciones al máximo posible (cooperativas de producción). Este tipo de empresas se rigen por una serie de principios o características que las hacen diferentes de las sociedades mercantiles o capitalistas (sociedades anónimas, limitadas, comanditarias, etc.), entre los que podemos

\footnotetext{
${ }^{1}$ En Mateos Ronco et al. (2011) puede verse un interesante estudio sobre los modelos de predicción del fracaso empresarial y su aplicabilidad en cooperativas agrarias. Puede completarse la revisión de la literatura en Veres et al. (2009) y Pozuelo et al. (2010).
} 
destacar el capital variable y su remuneración, el reparto del resultado en función de las operaciones realizadas por cada socio y la asignación obligatoria de parte de este resultado a la educación, formación y promoción de sus socios y trabajadores (Juste et al., 2011).

Estas características propias suponen realizar ciertas matizaciones en los ratios utilizados en el estudio estadístico, sobre todo en los de endeudamiento y en los de rentabilidad por las razones que vamos a exponer a continuación.

La libre adhesión y la consecuente variabilidad del capital social es una de las características que distingue a las sociedades cooperativas de las capitalistas. Así, una vez fijado en los estatutos de la cooperativa el capital social mínimo para que pueda funcionar, se pueden producir variaciones al alza por la entrada de nuevos socios o a la baja por la salida, siempre y cuando se respete la existencia de ese capital mínimo estatutario. Así, la anterior Orden ECO 3614/2003, de 16 de diciembre, de adaptación de la normativa contable a las peculiaridades de las cooperativas establecía que el capital de las cooperativas forma parte de los fondos propios, contabilizándose únicamente como una deuda en caso de baja del socio cooperativo. Sin embargo, dicha normativa fue objeto de revisión por parte del Instituto de Contabilidad y Auditoría de Cuentas (ICAC) para su adaptación al Plan General Contable de 2007 (PGC-2007) y a las Normas Internacionales de Información Financiera (NIIFs) mediante la Orden EHA/3360/2010, por la que se aprueban las normas sobre los aspectos contables de las sociedades cooperativas (NACSC), que entró en vigor el 1 de enero de 2011 y derogó la anterior Orden ECO. En estas nuevas NACSC se establece que, en general, las aportaciones de los socios y otros partícipes a las cooperativas, en la medida que no otorguen a la sociedad el derecho incondicional a rehusar su reembolso, deberán calificarse como pasivo. De esta forma, podemos establecer que en una cooperativa hay dos tipos de socios, los socios-propietarios y los socios-acreedores.

Sin embargo, entre la derogación de la anterior Orden ECO y la entrada en vigor de las nuevas NACSC, se estableció un período transitorio a partir de la entrada en vigor del nuevo PGC en 2008, en el cual, "los criterios por los que se establece la delimitación entre fondos propios y fondos ajenos de la Orden ECO podían seguir aplicándose hasta el 31 de diciembre de 2009, ampliándose este plazo un año más mediante el R.D. 2003/2009, es decir, hasta el 31 de diciembre de 2010.

Por lo tanto, dado que los ejercicios 2008, 2009 y 2010 forman parte del estudio estadístico, debemos tener en cuenta que en estos ejercicios el capital de las cooperativas puede haberse considerado como fondos propios en algunos casos y en otros, pensamos que los menos, haberse adaptado antes al cambio impulsado por las normas internacionales y haberse reclasificado en parte como fondos ajenos. 
En cuanto a la rentabilidad, teniendo en cuenta que la magnitud protagonista es el resultado, debemos tener claras las siguientes consideraciones en cuanto a su obtención por parte de las cooperativas.

En las sociedades cooperativas podemos distinguir dos tipos de resultados:

1. El derivado de las operaciones realizadas con los socios (excedente).

2. El derivado de las operaciones realizadas con terceros no socios y de operaciones extraordinarias.

El primero, es repartible entre los socios vía retorno cooperativo, una vez se han deducido las dotaciones al fondo de reserva obligatorio (FRO) y al fondo de educación, formación y promoción (FEFP), en proporción a las operaciones, actividades o servicios realizados por los socios con la cooperativa y nunca en función del capital como ocurre en las sociedades capitalistas. El resultado derivado de las operaciones con terceros no socios y, como mínimo, el 50\% de los beneficios extraordinarios, se destinarán también, una vez deducidas las pérdidas de ejercicios anteriores y antes de la consideración del impuesto sobre sociedades, a estos fondos. Por lo tanto, aunque equiparemos la dotación al FRO con la reserva legal de las denominadas sociedades capitalistas, debemos tener presente esa parte del resultado que no repercutirá directamente en los socios sino tal vez de forma indirecta según el uso que se haga del FEFP. Pero lo más importante sea tal vez que la verdadera rentabilidad de los socios no esté en función precisamente del reparto del excedente cooperativo sino de la obtención de mejores precios para sus productos y servicios a través de la actividad cooperativizada.

Por otro lado, para la obtención del resultado contable con las anteriores NACSC, en la cuenta de pérdidas y ganancias, después de obtener el resultado del ejercicio como en cualquier otra empresa, se incluían tres partidas más, propias de las cooperativas, para llegar al resultado cooperativo denominado excedente de la cooperativa. Los ingresos imputables al fondo de educación, formación y promoción (FEFP), la dotación a este mismo fondo y los intereses de las aportaciones al capital social y otros fondos, en la nueva normativa se integran en el formato normal de cualquier empresa, incorporando las dotaciones/ingresos del FEFP en un apartado del resultado de explotación (el 12) y los intereses como parte integrante de los gastos financieros, siempre y cuando estén preestablecidos. Así, a partir del ejercicio 2011 la obtención del resultado contable de las cooperativas sigue las mismas pautas que en el resto de empresas y no es diferente del excedente cooperativo. Este detalle es importante cuando se analizan y estudian las cooperativas por medio de bases de datos, ya que éstas utilizan el mismo formato para captar los datos de todas las empresas, sin tener en cuenta que en las cooperativas era diferente y que el resultado contable de la cuenta de pérdidas y ganancias no es lo mismo que el excedente de la cooperativa que aparece en los fondos propios del balance. 


\section{ESTUDIO ESTADÍSTICO}

Para elaborar un modelo de predicción de fracaso empresarial deberemos cubrir metodológicamente las siguientes etapas:

1. Definición de fracaso empresarial utilizada en el estudio.

2. Confección de una base de datos integrada por empresas cuyos registros cumplan las especificaciones exigidas por los objetivos del trabajo.

3. Selección y definición de las variables explicativas.

4. Selección de las técnicas de análisis y herramientas estadísticas.

\subsection{Concepto de fracaso empresarial utilizado en el estudio empírico: La variable dependiente}

La delimitación del concepto de fracaso empresarial es fundamental para la selección de las empresas que formarán las diferentes muestras sobre las que se realizará el estudio. Se ha optado por una definición de fracaso que permita distinguir con claridad las empresas sanas de las que no lo son. De esta manera se ha equiparado el fracaso a la calificación jurídica de situación concursal, según Ley Concursal 22/2003, de modo que una empresa se considerará fracasada si ha presentado un expediente concursal y sana en el caso contrario. Aunque este criterio $^{2}$, de marcado carácter jurídico, presenta algunos inconvenientes, como la reducción considerable de los tamaños muestrales, entendemos que son superados por las ventajas de objetividad y fijación de la fecha del fracaso que aporta en el proceso de selección de las empresas que integrarán las distintas muestras.

\subsection{Selección y fuentes de la muestra de empresas}

En el proceso de selección y obtención de las diferentes muestras de empresas se ha recurrido a la base de datos financieros SABI (Sistema de Análisis de Balances Ibéricos), cuyo uso ha sido cedido a la Universitat de València por la empresa INFORMA S.A.

\footnotetext{
${ }^{2}$ Otras definiciones que aparecen en la literatura sobre el fracaso empresarial son el incumplimiento en las obligaciones de pago, los descubiertos en cuentas bancarias, las pérdidas en ejercicios consecutivos, un patrimonio neto negativo, el cese en el reparto de dividendos, figurar en listados de morosos, cese de actividad, etc. En el sector cooperativo muchas veces el fracaso empresarial se manifiesta como cese de la actividad, significativas bajas de socios o el comienzo de procesos de reestructuración. Sin embargo, la consideración exclusiva de estas circunstancias nos hubiera impedido la elaboración de una base de datos de estudio que ofreciese resultados estadísticamente significativos. En Iturrioz (2010) e Iturrioz y Martín (2010) se detallan las peculiaridades del proceso concursal en las cooperativas y sus implicaciones financieras.
} 
Para seleccionar las empresas que formarían parte de la muestra de estimación el estudio se limitaría a aquellas cooperativas de naturaleza no financiera que habían fracasado, de acuerdo con la definición adoptada, entre los años 2008 y 2010. La selección efectuada, considerando esta condición, redujo la cifra a 42 empresas que habían presentado expedientes concursales en el período considerado.

En las 42 empresas resultantes se realizaron dos nuevos filtrados. Uno para que la muestra guardase un número proporcional al índice de fracaso del sector en que operaban y otro, por el que se descartaron aquellas empresas de reciente creación (hasta tres años) y las que no contenían datos contables completos de al menos tres ejercicios anteriores a la fecha del fracaso.

Tras estos procesos de selección y filtrado, el numero de firmas se redujo a 20 empresas cooperativas fracasadas que son las que definitivamente se integrarán en la muestra de estimación.

En la Tabla 1 se presenta el resumen de la distribución de la muestra de estimación de empresas quebradas por sectores de actividad económica mostrando el peso específico de cada grupo sectorial en el total de la muestra. La agrupación por sectores se ha realizado atendiendo al N.A.C.E. (rev) considerando los dos primeros dígitos de control, lo que ha permitido distinguir 10 actividades básicas.

Tabla 1

Distribución de la muestra de empresas quebradas por sectores de actividad

\begin{tabular}{|c|l|c|c|}
\hline NACE-Rev. & \multicolumn{1}{|c|}{ ACTIVIDAD } & $\begin{array}{c}\text { No } \\
\text { EMPRESAS }\end{array}$ & $\%$ \\
\hline 01 & Agricultura, ganadería, caza y servicios relacionados con las mismas & 2 & 10,00 \\
\hline 11 & Fabricación de bebidas & 2 & 10,00 \\
\hline 15 & Industria del cuero y del calzado & 1 & 5,00 \\
\hline 25 & Fabricación de productos metálicos, excepto maquinaria y equipo & 1 & 5,00 \\
\hline 41 & Construcción de edificios & 3 & 15,00 \\
\hline 43 & Actividades de construcción especializada & 3 & 15,00 \\
\hline 46 & $\begin{array}{l}\text { Comercio al por mayor e intermediarios del comercio, excepto de } \\
\text { vehículos de motor y motocicletas }\end{array}$ & 5 & 25,00 \\
\hline 47 & Comercio al por menor, excepto de vehículos de motor y motocicletas & 1 & 5,00 \\
\hline 52 & Almacenamiento y actividades anexas al transporte & 1 & 5,00 \\
\hline 80 & Actividades de seguridad e investigación & 1 & 5,00 \\
\hline TOTAL & & $\mathbf{2 0}$ & $\mathbf{1 0 0 , 0 0}$ \\
\hline
\end{tabular}

Fuente: Elaboración propia.

Para completar la muestra de estimación y poder aplicar ciertas herramientas estadísticas de clasificación, se recurrió a la técnica del emparejamiento consis- 
tente en asociar cada una de las empresas quebradas con una sana de las mismas características elegida aleatoriamente entre aquellas de un tamaño similar, medido en función del volumen de activo, y que operase en el mismo sector económico establecido por el N.A.C.E (rev). (Clasificación Nacional de Actividades Económicas) a nivel de cuatro dígitos y cuando no ha sido posible se ha descendido a tres dígitos. También se exigió que existiese una correspondencia temporal de sus estados contables con los ejercicios obtenidos para las empresas quebradas. Con este proceso se incorporaron otras 20 empresas quedando la muestra de estimación finalmente compuesta por 40 cooperativas, la mitad sanas y la otra mitad fracasadas.

A continuación, se plasmaron las observaciones de los tres años previos al momento del fracaso, ordenando los datos de forma que comenzaran en un momento común, al margen del año exacto al que hacían referencia. De esta forma el año (-1) sería el año anterior al fracaso, el año (-2) el correspondiente a dos años antes y así sucesivamente hasta el tercero. Esta ordenación de la información contable, según Gallego et al. (1997:5), "contribuye a que el modelo sea independiente de hechos específicos o de ciclos económicos subyacentes, aunque al combinar observaciones se asume implícitamente que las relaciones entre variables permanecen estacionarias en el tiempo".

\subsection{Selección y definición de las variables explicativas}

Uno de los aspectos más relevantes en la elaboración de modelos de predicción de fracaso empresarial es determinar las variables independientes que lo integrarán, en nuestro caso ratios económico-financieros. Como ya se ha argumentado, la primera dificultad para acometer esta etapa es la ausencia de una teoría general que guíe el proceso de selección, lo que constituye una fuerte limitación a la hora de modelizar el fracaso empresarial ${ }^{3}$. En este trabajo se procurará conciliar la experiencia aportada por otros autores con los objetivos propuestos. Por ello, para seleccionar los ratios con los que comenzaremos a trabajar nos basaremos en:

1. Ratios tradicionales en la literatura sobre análisis contable.

2. Frecuencia de aparición en estudios anteriores.

3. Facilidad para ser calculados y definidos en función de la información contable disponible.

Además, ninguno de los ratios considerados incorpora información de mercados bursátiles ya que ninguna de las cooperativas consideradas en las diferentes muestras cotizaba en el mercado de valores.

\footnotetext{
${ }^{3}$ Un estudio interesante que aporta una guía para la incorporación de ratios basados en un modelo económico-financiero de solvencia empresarial lo encontramos en Dieguez et al. (2006). También puede consultarse Labatut et al. (2009).
} 
En principio consideramos una amplia lista de ratios contenidos en diferentes categorías. Los solapamientos de información y problemas estadísticos, que necesariamente han de producirse, serán depurados con las técnicas estadísticas apropiadas eliminando aquellos que apenas aporten información adicional. Dado que nuestro objetivo es la formulación de modelos de predicción de fracaso empresarial, incidiremos en aquellas variables que, en principio, informen sobre los aspectos de la solvencia y rentabilidad de la firma, sin olvidar la influencia del endeudamiento. A estas categorías añadiremos las de rotación, actividad y estructura de activo. En esta selección de ratios no hemos incluido ratios definidos en términos de Cash-Flow operativo ya que en la revisión de los trabajos más relevantes no han mostrado una especial capacidad de clasificación. Describimos a continuación las categorías en las que incluiremos los diferentes ratios:

\section{Rentabilidad (REN)}

Genéricamente la rentabilidad se identifica con el cociente entre beneficio e inversión y es la tasa con que la empresa remunera al capital empleado. La rentabilidad es una medida del rendimiento empresarial. Dependiendo del tipo de beneficio específico considerado, que figurará en el numerador de los ratios propuestos y de la magnitud que lo ha generado, representada en el denominador, tendremos diversos tipos de rentabilidad, económica y financiera. Existe prácticamente unanimidad en la doctrina de análisis contable en considerar la rentabilidad económica como indicador de la eficacia de la empresa en la utilización de sus inversiones y la rentabilidad financiera como indicador de la capacidad de la empresa para crear riqueza a favor de sus propietarios. Sin embargo, no debemos olvidar que en las cooperativas la mejor rentabilidad de sus socios vendrá probablemente por la obtención de unos buenos precios de los productos y servicios cooperativizados. Dentro de la categoría de rentabilidad hemos considerado unos ratios de naturaleza mixta integrados por las magnitudes de beneficio en sus distintos niveles y de endeudamiento. También, en la categoría de rentabilidad se han integrado algunos ratios planteados con el Cash-Flow en su acepción tradicional de recursos generados, es decir, beneficio + amortizaciones + provisiones.

\section{Estructura financiera o Endeudamiento (EF)}

La estructura financiera informa sobre el origen y composición de los recursos financieros que han servido para llevar a cabo la inversión que se ha materializado en el conjunto de factores de producción a disposición de la empresa o estructura económica. Pero tal y como hemos indicado en las especificidades de las cooperativas, nos encontramos con una magnitud, el capital, que puede haberse considerado patrimonio neto cuando en el fondo puede ser un pasivo, al menos en parte. 
Con estos indicadores se podrá establecer el grado de dependencia de la empresa en función de la fuente de recursos financieros que utiliza.

\section{Actividad (ACT)}

Con los regresores de actividad se trata de ponderar cada concepto de gasto respecto a la actividad realizada. Podremos plantear este tipo de ratios atendiendo a la clasificación de los gastos según su variabilidad en relación con la actividad, considerando los gastos fijos y variables o también, atendiendo a la clasificación funcional de los gastos.

\section{4. $\underline{\text { Rotación }}$ (ROT)}

Con ellos se pretenderá determinar el grado de eficiencia de las operaciones más significativas de la empresa, fundamentalmente aquellas vinculadas con la actividad principal. A diferencia de los ratios de rentabilidad, con los que se pretende determinar el grado de efectividad general, con los de rotación se pretende determinar el grado de efectividad o de dinamismo de activos específicos. En la mayoría de estos ratios figurará en el numerador la cifra de ventas y en el denominador el saldo del activo cuya efectividad se pretende medir.

\section{Solvencia a corto plazo (SOLV)}

Los ratios de solvencia a corto plazo o liquidez, ligados estrechamente a la situación de equilibrio financiero, indican la capacidad que la empresa tiene para atender sus deudas a corto plazo (por corto plazo entenderemos el ejercicio económico o el ciclo de explotación si este es superior a un año). Estos ratios relacionan partidas o masas patrimoniales de la estructura circulante de la empresa, tanto del activo como del pasivo. Nos servirán para determinar la liquidez del activo.

\section{Solvencia a largo plazo (SOLV LP)}

Con estos ratios se pretende determinar la capacidad de la empresa para afrontar sus deudas a largo plazo. Estas medidas centran su atención en la estructura financiera y operativa de la empresa contemplada desde el largo plazo.

\section{Estructura económica (CF)}

Los ratios de esta categoría relacionan elementos y masas patrimoniales de activo mostrando la estructura de las inversiones ponderando cada elemento o partida respecto al total de ellas. Al ser muy numerosos los elementos de la estructura económica se puede plantear un numero considerable de ratios de esta categoría.

En nuestro estudio partiremos de la información contenida en los estados financieros de las sociedades cooperativas transformándola en ratios, lo que nos permitirá establecer con rigor su situación económico-financiera y realizar 
comparaciones entre las distintas empresas y diferentes periodos económicos. Por razones de tipo operativo no hemos considerado información de distinto origen, como la de naturaleza cualitativa.

La lista de los ratios considerados inicialmente y separados por categorías la mostramos en la Tabla 2:

Tabla 2

Ratios utilizados en el estudio empírico

\begin{tabular}{|c|c|c|}
\hline \multicolumn{3}{|c|}{ RENTABILIDAD } \\
\hline CLAVE & RATIO & \\
\hline REN 1 & RESULTADO DE EXPLOTACION/ACTIVO & $\mathrm{RE} / \mathrm{A}$ \\
\hline REN 2 & RESULTADO DEL EJERCICIO/ACTIVO & $\mathrm{R} / \mathrm{A}$ \\
\hline REN 3 & RESULTADO DEL EJERCICIO/NETO & $\mathrm{R} / \mathrm{N}$ \\
\hline REN 4 & RESULTADO DEL EJERCICIO/ PASIVO & $\mathrm{R} / \mathrm{P}$ \\
\hline REN 5 & RESULTADO DE ACTIVIDADES ORDINARIAS $\left({ }^{*}\right) / A C T I V O$ & $\mathrm{RO} / \mathrm{A}$ \\
\hline REN 6 & RESULTADO DE EXPLOTACION/VENTAS & $\mathrm{RE} / \mathrm{V}$ \\
\hline REN 7 & RESULTADO DE ACTIV. ORDINARIAS/PASIVO & $\mathrm{RO} / \mathrm{P}$ \\
\hline REN 8 & RESULTADO DE ACTIV. ORDINARIAS/NETO & $\mathrm{RO} / \mathrm{N}$ \\
\hline REN 9 & RESULTADO DEL EJERCICIO/VENTAS & $\mathrm{R} / \mathrm{V}$ \\
\hline REN 10 & CASH FLOW RECURSOS GENERADOS/NETO & $\mathrm{CF} / \mathrm{N}$ \\
\hline REN 11 & CASH FLOW RECURSOS GENERADOS/ACTIVO & CF/A \\
\hline REN 12 & CASH FLOW RECURSOS GENERADOS/PASIVO & $\mathrm{CF} / \mathrm{P}$ \\
\hline REN 13 & CASH FLOW RECURSOS GENER./PASIVO CORRIENTE & CF/PC \\
\hline REN 14 & RESULTADO ANTES DE IMPUESTOS/ ACTIVO & $\mathrm{RAI} / \mathrm{A}$ \\
\hline REN 15 & CASH FLOW RECURSOS GENERADOS/VENTAS & CF/V \\
\hline REN 16 & RESULTADO DE EXPLOTACION/GASTOS FROS. & RE/GF \\
\hline REN 17 & RESULTADO ANTES DE IMPUESTOS/ NETO & $\mathrm{RAI} / \mathrm{N}$ \\
\hline REN 19 & RESULTADO ANTES DE IMPUESTOS/ VENTAS & $\mathrm{RAI} / \mathrm{V}$ \\
\hline \multicolumn{3}{|c|}{$\left(^{*}\right)$ El resultado de actividades ordinarias es considerado antes de impuestos } \\
\hline \multicolumn{3}{|c|}{ ESTRUCTURA FINANCIERA } \\
\hline CLAVE & RATIO & \\
\hline EF 1 & PASIVO/NETO & $\mathrm{P} / \mathrm{N}$ \\
\hline EF 2 & PASIVO NO CORRIENTE/NETO & $\mathrm{PNC/N}$ \\
\hline EF 3 & PASIVO CORRIENTE/NETO & $\mathrm{PC} / \mathrm{N}$ \\
\hline EF 4 & GASTOS FINANCIEROS/PASIVO & GF/P \\
\hline EF 5 & PASIVO CORRIENTE/PASIVO & $\mathrm{PC} / \mathrm{P}$ \\
\hline EF 6 & GASTOS FINANCIEROS/VENTAS & GF/N \\
\hline \multicolumn{3}{|l|}{ ACTIVIDAD } \\
\hline CLAVE & RATIO & \\
\hline ACT 1 & VALOR AÑADIDO/VENTAS & VA/V \\
\hline ACT 2 & VALOR AÑADIDO/GASTOS DE PERSONAL & GP/VA \\
\hline ACT 3 & VALOR AÑADIDO/GASTOS FINANCIEROS & GF/VA \\
\hline ACT 4 & RESULTADO DEL EJERCICIO/VALOR AÑADIDO & R/VA \\
\hline ACT 5 & GASTOS DE PERSONAL/VENTAS & GP/N \\
\hline
\end{tabular}


Tabla 2 (continuación)

Ratios utilizados en el estudio empírico

\begin{tabular}{|c|c|c|}
\hline \multicolumn{3}{|l|}{ ROTACIÓN } \\
\hline CLAVE & RATIO & \\
\hline ROT 1 & VENTAS/ACTIVO & VIA \\
\hline ROT 2 & VENTAS/ACTIVO CORRIENTE & VIAC \\
\hline ROT 3 & VENTAS/ACTIVO NO CORRIENTE & VIANC \\
\hline ROT 4 & VENTAS/NETO + PASIVO NO CORRIENTE & $\mathrm{V} / \mathrm{N}+\mathrm{PNC}$ \\
\hline ROT 5 & VENTAS/PASIVO CORRIENTE & V/PC \\
\hline ROT 6 & ACTIVO/INGRESOS DE EXPLOTACION & $\mathrm{A} / \mathrm{IE}$ \\
\hline ROT 7 & VENTAS/EXISTENCIAS & V/EX \\
\hline \multicolumn{3}{|c|}{ SOLVENCIA (Liquidez) } \\
\hline CLAVE & RATIO & \\
\hline SOLV 1 & ACTIVO CORRIENTE/PASIVO CORRIENTE & $\mathrm{AC} / \mathrm{PC}$ \\
\hline SOLV 2 & ACTIVO CORRIENTE - EXISTENCIAS/PASIVO CORRIENTE & AC-EX/PC \\
\hline SOLV 3 & DISPONIBLE/PASIVO CORRIENTE & $\mathrm{D} / \mathrm{PC}$ \\
\hline SOLV 4 & CAPITAL CIRCULANTE/PASIVO CORRIENTE & $\mathrm{CC} / \mathrm{PC}$ \\
\hline SOLV 5 & CAPITAL CIRCULANTE/ACTIVO & $\mathrm{CC} / \mathrm{A}$ \\
\hline SOLV 6 & CAPITAL CIRCULANTE/VENTAS & $\mathrm{CC} / \mathrm{V}$ \\
\hline SOLV 7 & RECURSOS GENER. ANTES DE IMPUESTOS/PAS. CORR. & RGAI/PC \\
\hline \multicolumn{3}{|c|}{ SOLVENCIA A LARGO PLAZO } \\
\hline CLAVE & RATIO & \\
\hline SOLV LP 1 & ACTIVO NO CORRIENTE/NETO & ANC/N \\
\hline SOLV LP 2 & REC. GENER. ANTES DE IMPUESTOS/PASIVO & RGAI/P \\
\hline SOLV LP 3 & ACTIVO /PASIVO & $\mathrm{A} / \mathrm{P}$ \\
\hline \multicolumn{3}{|c|}{ ESTRUCTURA ECONÓMICA } \\
\hline CLAVE & RATIO & \\
\hline CF 1 & EXISTENCIAS/ACTIVO CORRIENTE & EX/AC \\
\hline $\mathrm{CF} 2$ & ACTIVO CORRIENTE/ACTIVO & $\mathrm{AC} / \mathrm{A}$ \\
\hline CF 3 & ACTIVO CORRIENTE/ACTIVO NO CORRIENTE & AC/ANC \\
\hline CF 4 & EXISTENCIAS/CAPITAL CIRCULANTE & $\mathrm{EX} / \mathrm{CC}$ \\
\hline CF 5 & EXISTENCIAS/ACTIVO & $\mathrm{E} / \mathrm{A}$ \\
\hline CF 6 & REALIZABLE/ ACTIVO & $\mathrm{REA} / \mathrm{A}$ \\
\hline CF 7 & DISPONIBLE/ ACTIVO & $\mathrm{D} / \mathrm{A}$ \\
\hline
\end{tabular}

Fuente: Elaboración propia.

Todas las partidas integrantes de los ratios han sido derivadas del Balance de Situación y cuenta de Pérdidas y Ganancias de las empresas que componen las diferentes muestras.

\subsection{Metodología estadística}

Para la realización del estudio empírico nos hemos apoyado en un modelo de predicción del fracaso empresarial basado en la Regresión Logística Binaria (RLB), esto es, un tipo de función que explica el comportamiento del fracaso como variable dependiente binaria, con la que en anteriores trabajos se ha obtenido muy buenos resultados. Para llevar a cabo el análisis estadístico se ha recu- 
rrido al programa STATA 11.2 (Statistics. Data Analysis) de Windows, al que se exportaron los datos de las empresas disponibles en la base de datos $S A B I$.

La construcción de los modelos de predicción del fracaso de las sociedades cooperativas se ha emprendido desde una óptica multivariante, es decir, de acuerdo con la información proporcionada por un conjunto de variables exógenas o independientes (Rodríguez y Barrios, 2003; y Alfonso et al., 2004). Las variables que se han considerado en el modelo de la RLB como instrumento de predicción del fracaso son:

- Variable dependiente: es una variable dicotómica que toma valor 1 cuando se trata de una sociedad cooperativa que haya fracasado; y que toma valor 0 cuando no haya fracasado. Tomará la notación de $F_{n}$, siendo $n$ el número de años anteriores al de referencia de la quiebra.

- Variables independientes: ratios económico-financieros de las sociedades cooperativas. La ponderación de estos ratios con sus respectivos coeficientes ofrece como resultado una puntuación o probabilidad de que tenga lugar el fracaso de la sociedad cooperativa. Como notación de estas variables seguiremos la empleada para los ratios considerados de la Tabla 2.

Los resultados que se alcancen permitirán obtener una visión de las empresas cooperativas que ayude a precisar en que áreas se generan las situaciones de desequilibrio que la pueden llevar al fracaso.

Con anterioridad a la aplicación de la metodología basada en la RLB, resulta imprescindible la reducción de la lista de ratios o posibles variables dependientes de la que partimos, seleccionando solamente aquellos que muestren capacidad de añadir globalmente información no redundante. De este modo intentamos evitar los problemas y distorsiones en los resultados derivados de la presencia de multicolinealidad. Para ello hemos utilizado el análisis factorial de componentes principales (AFCP), pues proporciona resultados muy satisfactorios en cuanto a la reducción del conjunto inicial de variables y es compatible con los problemas subyacentes que la falta de normalidad de la distribución de las variables provoca en el procedimiento.

La técnica del AFCP facilita la identificación de los componentes (factores) que sucesivamente explican la mayor parte de la varianza total. Esto es, proporciona combinaciones lineales de las variables originales que contienen la mayor proporción de la variación total. En el AFCP el primer factor o componente es aquel que explica una mayor parte de la varianza total, el segundo factor el que explica la mayor parte de la varianza restante, es decir, de la no explicada por el primero y así sucesivamente. En definitiva, el AFCP permite la reducción de las variables de partida, revelando la mayor cantidad posible de la varianza contenida en los indicadores con el menor número posible de factores. 
El análisis factorial AFCP se ha efectuado mediante el comando pca (Principal component analysis) del programa informático STATA 11.2. Asimismo, nos hemos valido del comando rotate para obtener las rotaciones ortogonales varimax y así extraer la solución factorial más plausible, es decir, la mejor agrupación de las variables o indicadores (ratios económico-financieros considerados) en factores o componentes. Todo el proceso descrito se ha realizado por separado para cada uno de los años estudiados.

Una vez se han identificado los ratios económico-financieros con una mayor carga explicativa en el AFCP se ha comprobado mediante la matriz de correlaciones que no hubiera problemas de multicolinealidad y, en caso afirmativo, se han eliminado las variables con una correlación más alta. A continuación, se ha realizado el proceso de modelización mediante la estimación de modelos de predicción aplicando como ya se ha indicado la técnica de la RLB. Se ha planteado un modelo para cada uno de los tres ejercicios previos al fracaso. Los aspectos más relevantes de los modelos resultantes pasamos a comentarlos a continuación, tratando de descifrar y describir globalmente las circunstancias que motivaron a las empresas cooperativas consideradas a entrar en procesos de crisis y lo que supuso, en la mayoría de los casos, su posterior liquidación.

Hay que señalar que todo el proceso de modelización se ha efectuado considerando una probabilidad previa de pertenencia a cada grupo del $50 \%$, ya que en la muestra de empresas hay el mismo número de empresas sanas que de fracasadas, lo que supone que este punto de corte minimiza el valor medio esperado de los errores de clasificación. Estamos, por tanto, considerando que el coste de predecir una empresa quebrada como sana (error tipo I) es similar al coste de predecir una empresa sana como quebrada (error tipo II).

\section{$\underline{1}^{\underline{e r}} \underline{\text { año anterior al fracaso }}$}

La Tabla 3 presenta los ratios económico-financieros identificados mediante el AFCP y que entrarían en el proceso de modelización de las sociedades cooperativas para el año anterior a la situación del fracaso. Se han elegido solamente aquellos factores/componentes con un valor propio superior a la unidad, es decir con una mayor contribución a la explicación de la varianza total. Y de estos factores solamente se han considerado los ratios cuyo peso dentro del factor sea en valor absoluto superior a 0,8 . Los datos se han ordenado en función de su contribución a la varianza explicada.

La aplicación del análisis factorial nos ha permitido reducir considerablemente el número de indicadores iniciales, pasando de una lista inicial de 53 ratios a considerar 29 componentes o factores para el primer año anterior a la situación de quiebra de la sociedad cooperativa. En general, en todos los años analizados los factores de mayor contribución informativa son los que incluyen las variables de rentabilidad. El predominio de los ratios de esta categoría se ve 
favorecido por el amplio abanico de posibilidades que se han tenido en cuenta para su formulación. A continuación, destacan los ratios de solvencia a corto plazo o liquidez y los de rotación.

Tabla 3

Ratios con $p>0$ '8 o con mayor peso en cada factor de valor propio $>1$. Año $n-1$

\begin{tabular}{|c|c|c|c|c|c|c|}
\hline Comp1 & Comp2 & Comp3 & Comp4 & Comp5 & Comp6 & Comp7 \\
\hline REN1 & ROT1 & REN3 & SOLV1 & ACT5 & REN16 & ACT4 \\
REN2 & ROT2 & REN8 & SOLV2 & CF6 & ACT3 & SOLV5 \\
REN5 & ROT3 & REN10 & SOLV3 & EF4 & SOLVLP2 & CF5 \\
ACT2 & ROT4 & REN17 & SOLVLP3 & & & \\
& EF1 & & CF3 & & & \\
& EF3 & & CF7 & & & \\
\hline
\end{tabular}

Fuente: Elaboración propia.

Antes de realizar la RLB hemos comprobado mediante la matriz de correlaciones que no hubiese problemas de multicolinealidad entre las variables de la Tabla 3. Una correlación alta entre las variables podría conducir a una interpretación errónea de los resultados de la regresión (coeficientes inexactos, niveles de significación estadísticos equivocados e incluso signos incorrectos) y por tanto es necesario analizar la posible gravedad del problema. La matriz de correlaciones de las variables independientes, que no se incluye por motivos de espacio, indica correlaciones muy altas para las variables REN8, REN10, REN17, SOLV3, ROT2 y EF3, y por tanto se han eliminado y no se han considerado en la RLB. El resto de correlaciones son muy bajas. Judge et al. (1988) y Gujarati (1995) afirman que las correlaciones inferiores a 0,80 en valor absoluto no resultarían demasiado perjudiciales en lo que respecta a la multicolinealidad. Por otra parte, Hosmer y Lemeshow (1989) sostienen que todas las variables en teoría relevantes deberían incluirse en el modelo de regresión logística a menos que su colinealidad sea extrema.

Las estimaciones multivariantes realizadas sobre los datos de las sociedades cooperativas para el primer año anterior al fracaso ofrecen como resultado una función compuesta por dos variables que muestran las áreas donde se producen los desequilibrios más relevantes en estas sociedades (véase Tabla 4 de la RLB).

$$
\mathrm{F}_{1}=1,1194+1,2655 \mathrm{REN} 2-22,5972 \mathrm{SOLVLP} 2+\varepsilon
$$

En la totalidad de las estimaciones realizadas el punto de corte se ha establecido en 0,5 . Por debajo de este valor, $\mathrm{F}_{1}<0,5$, las empresas son clasificadas como sanas y por encima, $F_{1}>0,5$, como fracasadas.

De forma global los ratios que conforman el modelo obtenido ejercen un efecto significativo en la separación de los dos grupos de sociedades cooperativas, sanas y fracasadas, alcanzando un porcentaje global de clasificación co- 
rrecta del $85,00 \%$. Los valores $R^{2}$ no se facilitan pues el uso de medidas de la bondad del ajuste en el análisis de regresión logístico es un tema de debate aún no resuelto (Craswell, 1999). Además, Veall y Zimmermann (1994) sugieren que el valor de $R^{2}$ tiende a ser sustancialmente menor que el obtenido en la regresión lineal simple. Se proporciona, sin embargo, el valor del estadístico ${ }^{4}$ Pseudo $R^{2}$.

Tabla 4

Regresión logística. Ejercicio 1 previo al fracaso

\begin{tabular}{lrrrrr}
\hline \multicolumn{1}{c}{ Variable } & \multicolumn{1}{c}{ Beta } & Error típico & Wald & \multicolumn{1}{c}{ Sig. } & \multicolumn{1}{c}{$\operatorname{Exp(B)}$} \\
\hline REN2 & 1,2655 & 0,7009 & 1,81 & 0,071 & 3,5450 \\
SOLVLP2 & $-22,5972$ & 8,6434 & $-2,61$ & 0,009 & $1,54 \mathrm{e}-10$ \\
Constante & 1,1194 & 0,5669 & 1,97 & 0,048 &. \\
\hline
\end{tabular}

Notas: REN2: resultado del ejercicio dividido entre el activo. SOLVLP2: recursos generados antes de impuestos dividido entre pasivo. Tamaño muestral 40; Chi cuadrado 19,73; $p=0,000$; Pseudo $R^{2}$ 35,59; Clasificación correcta $85,00 \%$.

Fuente: Elaboración propia.

El modelo estimado globalmente es significativo. Se rechaza la hipótesis nula de que todos los coeficientes de la función sean nulos al alcanzar la Chi cuadrado un valor de 19,73 y nivel de significación critico 0,000. También los distintos coeficientes considerados uno a uno son significativamente distintos de cero, pues el nivel de significación del estadístico de Wald permite rechazar la hipótesis nula con un error inferior a un 0,07 . De acuerdo con el signo negativo del ratio SOLVLP2 se observa claramente que cuantos mayores sean los recursos generados por una sociedad cooperativa la posibilidad de que entre en una situación concursal es menor. Sin embargo, llama la atención el signo positivo que alcanza la variable $R E N 2$, lo que podemos interpretar como que ante una situación financiera límite de la cooperativa, dada su inminente situación concursal, es muy probable que las solicitudes de baja por parte de los socios se multipliquen, con la consiguiente reducción de recursos financieros, lo que se podría tratar de compensar con una disminución de la remuneración de los socios vía precios de liquidación, tratando de obtener un mayor resultado contable que permita paliar esa reducción de recursos financieros por la vía de la autofinanciación.

\footnotetext{
${ }^{4}$ La regresión logística no tiene un equivalente al $R^{2}$ de la regresión por mínimos cuadrados, sin embargo, muchos investigadores han tratado de obtener uno que se le aproxime. Hay una amplia variedad de estadísticos pseudo $R^{2}$ que puede dar lugar a conclusiones contradictorias. Debido a que el estadístico pseudo $R^{2}$ no significa lo mismo que el $R^{2}$ de la regresión por mínimos cuadrados (la proporción de la varianza para la variable de respuesta explicada por los predictores), se sugiere mucha cautela en su interpretación (Introduction to SAS, 2012).
} 
Una interpretación interesante de los coeficientes en una regresión logística se refiere al cambio en el logaritmo de las posibilidades de la variable dependiente dado un cambio unitario en la variable independiente (Sharma y Sidhu, 2001). El exponente del coeficiente representa la ratio de cambio en las posibilidades del evento analizado ante un cambio de una unidad en el factor de predicción (variables independientes). Este exponente origina el cambio del multiplicador estimado en las posibilidades de ocurrencia de la variable dependiente, y $100 *$ [Exp (B) - 1] recoge el porcentaje de cambio en las posibilidades de la variable dependiente dada una unidad de cambio en el coeficiente (Demaris, 1992).

La Tabla 5 muestra el desglose global de la clasificación correcta del modelo. El porcentaje de aciertos en sociedades cooperativas fracasadas es del $90,00 \%$ y el de aciertos en sociedades cooperativas sanas del 80,0\% Unos porcentajes que consideramos muy satisfactorios y que permitirían, en su caso, la aplicación de este modelo a contextos financieros para la valoración de los riesgos, en los que el coste de clasificación errónea de una sociedad cooperativa sana como quebrada es superior al de clasificar una fracasada como sana.

Tabla 5

Clasificación correcta. Ejercicio 1 previo al fracaso

\begin{tabular}{|c|c|c|c|c|}
\hline \multirow{3}{*}{\multicolumn{2}{|c|}{ Pronosticado }} & \multicolumn{3}{|c|}{ Observado } \\
\hline & & \multicolumn{2}{|c|}{ Fracasada } & \multirow[b]{2}{*}{ Total } \\
\hline & & Si & No & \\
\hline \multirow{2}{*}{ Fracasada } & $\mathrm{Si}$ & 18 & 4 & 22 \\
\hline & No & 2 & 16 & 18 \\
\hline \multicolumn{2}{|c|}{ Porcentaje correcto } & $90,00 \%$ & $80,00 \%$ & $85,00 \%$ \\
\hline
\end{tabular}

Fuente: Elaboración propia.

\section{$\underline{2^{\circ} \text { año anterior al fracaso }}$}

La Tabla 6 recoge los ratios económico-financieros identificados mediante el AFCP y que entrarán en el proceso de modelización de las sociedades cooperativas dos años previos a la situación del fracaso.

Mediante el análisis factorial hemos reducido el número de indicadores iniciales, pasando de una lista inicial de 53 ratios a considerar 27 componentes o factores para el segundo año anterior a la situación de quiebra de la sociedad cooperativa. En general, en todos los años analizados los factores de mayor contribución informativa son los que incluyen las variables de rentabilidad. El 
predominio de los ratios de esta categoría se ve favorecido por el amplio abanico de posibilidades que se han tenido en cuenta para su formulación. A continuación, destacan los ratios de solvencia a corto plazo o liquidez y los de endeudamiento.

\section{Tabla 6}

Ratios con $p>0$ '8 o con mayor peso en cada factor de valor propio > 1. Año n-2

\begin{tabular}{|c|c|c|c|c|c|c|}
\hline Comp1 & Comp2 & Comp3 & Comp4 & Comp5 & Comp6 & Comp7 \\
\hline REN2 & SOLV1 & SOLVLP1 & SOLV4 & REN16 & REN3 & SOLV6 \\
REN5 & SOLV2 & ROT1 & CF1 & ACT3 & REN8 & ROT6 \\
& SOLV3 & ROT2 & CF4 & & REN10 & EF2 \\
& CF3 & ROT4 & CF5 & & REN17 & EF6 \\
& CF7 & EF1 & & & & \\
& & EF3 & & & & \\
\hline
\end{tabular}

Fuente: Elaboración propia.

La matriz de correlaciones ${ }^{5}$ de las variables de la Tabla 6 ha permitido identificar problemas de multicolinealidad, lo que nos ha obligado a eliminar las siguientes variables: REN5, REN8, REN10, REN17, SOLV3, ACT3, ROT2, EF2, EF3 y CF5.

Los regresores que forman la función logit derivada para el segundo año antes del fracaso (Tabla 7), permiten apreciar que las áreas donde se producen los desequilibrios, reveladoras del fracaso empresarial, se localizan en la capacidad para generar recursos y en los costes de endeudamiento. La función que define el fracaso quedaría formulada de la siguiente manera:

$$
\mathrm{F}_{2}=-0,7575-42,4519 \mathrm{REN} 2+66,396 \mathrm{EF} 6+\varepsilon
$$

Tabla 7

Regresión logística. Ejercicio 2 previo al fracaso

\begin{tabular}{lcrrrc}
\hline \multicolumn{1}{c}{ Variable } & Beta & Error típico & Wald & Sig. & Exp(B) \\
\hline REN2 & $-42,4519$ & 18,8365 & $-2,25$ & 0,024 & $3,66 \mathrm{e}-19$ \\
EF6 & 66,3960 & 28,1912 & 2,36 & 0,019 & $6,85 \mathrm{e}+28$ \\
Constante & $-0,7575$ & 0,6304 & $-1,20$ & 0,229 &. \\
\hline
\end{tabular}

Notas: REN2: resultado del ejercicio dividido entre activo total. EF6: gastos financieros dividido entre ventas. Tamaño muestral 40; Chi cuadrado 24,57; $p=0,000$; Pseudo $R^{2} 44,31$; Clasificación correcta 77,5.

Fuente: Elaboración propia.

\footnotetext{
${ }^{5}$ No hemos incluido en el trabajo ninguna matriz de correlaciones por motivos de espacio.
} 
Esta función está formada por dos regresores, lo que nos sugiere que dos años antes de producirse el fracaso sus señales ya son claramente perceptibles, al menos en las áreas representadas por estas variables.

El signo negativo del coeficiente de la variable REN2 indica que cuanto mayor sea el resultado del ejercicio la posibilidad de que una sociedad cooperativa quiebre es menor. Y el signo positivo del coeficiente de la variable EF6 recoge la posibilidad de una situación concursal en este tipo de sociedades incrementa a medida que aumentan los gastos financieros, motivados por un mayor endeudamiento.

Tabla 8

Clasificación correcta. Ejercicio 2 previo al fracaso

\begin{tabular}{|c|c|c|c|c|}
\hline \multirow{3}{*}{\multicolumn{2}{|c|}{ Pronosticado }} & \multicolumn{3}{|c|}{ Observado } \\
\hline & & \multicolumn{2}{|c|}{ Fracasada } & \multirow[b]{2}{*}{ Total } \\
\hline & & Si & No & \\
\hline \multirow{2}{*}{ Fracasada } & $\mathrm{Si}$ & 14 & 3 & 17 \\
\hline & No & 6 & 17 & 23 \\
\hline \multicolumn{2}{|c|}{ Porcentaje correcto } & $70,00 \%$ & $85,00 \%$ & $77,50 \%$ \\
\hline
\end{tabular}

Fuente: Elaboración propia.

Globalmente el modelo es significativo como lo constata el valor de la Chi cuadrado que alcanza un valor de 24,57 con un nivel de significatividad crítico del 0,000. Por tanto, rechazamos la hipótesis nula de que los coeficientes son nulos. Los valores individuales del estadístico de Wald hacen posible rechazar la hipótesis nula de que los coeficientes son iguales a cero con un error inferior al 0,05 .

La tabla de clasificación correcta del ejercicio 2 (Tabla 8) previo al fracaso de las sociedades cooperativas muestra que el modelo consigue un $77,50 \%$ de aciertos totales de clasificación. El porcentaje de aciertos en sociedades cooperativas fracasadas es del $70,00 \%$ y el de aciertos en sociedades cooperativas sanas del $85,00 \%$. Por consiguiente, los resultados alcanzados en el proceso de validación son muy aceptables.

\section{$\underline{3}^{\text {er }}$ año anterior al fracaso}

Los ratios con mayor peso en cada factor de valor propio para el tercer año antes del fracaso se detallan en la Tabla 9. Como ya se ha indicado se han considerado solamente los ratios cuyo peso dentro del factor sea en valor absoluto 
superior a 0,8 y los factores o componentes elegidos tienen un valor propio superior a la unidad.

Tabla 9

Ratios con $p>0$ '8 o con mayor peso en cada factor de valor propio > 1. Año n-3

\begin{tabular}{|c|c|c|c|c|c|c|}
\hline Comp1 & Comp2 & Comp3 & Comp4 & Comp5 & Comp6 & Comp7 \\
\hline REN5 & REN3 & ROT3 & ACT3 & CF1 & SOLV1 & SOLV5 \\
REN11 & REN8 & CF2 & ROT1 & CF4 & SOLV2 & ACT1 \\
ACT2 & REN10 & CF3 & ROT2 & CF5 & SOLV4 & ACT5 \\
& REN17 & CF7 & ROT4 & & ROT5 & CF6 \\
& & & & & & EF4 \\
\hline
\end{tabular}

Fuente: Elaboración propia.

La lista inicial de 53 ratios ha quedado reducida a 26 factores o componentes, para el tercer año anterior a la situación de quiebra de la sociedad cooperativa. En general, en todos los años analizados los factores de mayor contribución informativa son los que incluyen las variables de rentabilidad. El predominio de los ratios de esta categoría se ve favorecido por el amplio abanico de posibilidades que se han tenido en cuenta para su formulación. A continuación, destacan los ratios de solvencia a corto plazo o liquidez y los de endeudamiento.

La matriz de correlaciones de las variables de la Tabla 9 nos ha permitido identificar aquellas variables con correlaciones muy altas, las cuales han sido eliminadas para evitar problemas de multicolinealidad: REN8, REN10, REN17, SOLV2, ROT2, CF4 y CF5.

El modelo obtenido para el tercer año antes del fracaso ha quedado formulado como sigue (véase Tabla 10):

$$
\mathrm{F}_{3}=1,2544-9,4619 \mathrm{CF} 7-0,0287 \mathrm{ROT} 4+\varepsilon
$$

Tabla 10

Regresión logística. Ejercicio 3 previo al fracaso

\begin{tabular}{llcccc}
\hline \multicolumn{1}{c}{ Variable } & Beta & Error típico & Wald & Sig. & Exp(B) \\
\hline CF7 & $-9,4619$ & 3,9844 & $-2,37$ & 0,018 & 0,0001 \\
ROT4 & $-0,0287$ & 0,0261 & -1.10 & 0.271 & 0,9716 \\
Constante & 1,2544 & 0,5604 & 2.24 & 0,025 &. \\
\hline
\end{tabular}

Notas: CF7: disponible dividido entre el activo total. ROT4: ventas dividido entre la suma de fondos propios y pasivo fijo. Tamaño muestral 40; Chi cuadrado 11,07; $p=0,003$; Pseudo $R^{2}$ 19,97; Clasificación correcta 72,50.

Fuente: Elaboración propia. 
El modelo globalmente es significativo, como lo constata que la Chi cuadrado alcance un valor de 11,07 y un nivel de significatividad crítico de 0,00 , lo que nos permite rechazar la hipótesis nula de que los coeficientes de la función son nulos. Considerando los diferentes coeficientes por separado también es significativamente distinto de cero el correspondiente a $C F 7$, a un nivel de significación del 5\%. El signo negativo del coeficiente de esta variable muestra que cuanto mayor sea el disponible la posibilidad de que una sociedad cooperativa quiebre es menor.

Tabla 11

Clasificación correcta. Ejercicio 3 previo al fracaso

\begin{tabular}{|c|c|c|c|c|}
\hline \multirow{3}{*}{\multicolumn{2}{|c|}{ Pronosticado }} & \multicolumn{3}{|c|}{ Observado } \\
\hline & & \multicolumn{2}{|c|}{ Fracasada } & \multirow[b]{2}{*}{ Total } \\
\hline & & Si & No & \\
\hline \multirow{2}{*}{ Fracasada } & $\mathrm{Si}$ & 16 & 7 & 23 \\
\hline & No & 4 & 13 & 17 \\
\hline \multicolumn{2}{|c|}{ Porcentaje correcto } & $80,00 \%$ & $65,00 \%$ & $72,50 \%$ \\
\hline
\end{tabular}

Fuente: Elaboración propia.

El modelo resultante está formado por dos variables con las que consigue un grado de acierto global en la clasificación del $72,50 \%$, y con un porcentaje de aciertos en sociedades cooperativas fracasadas del $80,00 \%$ y de aciertos en sociedades cooperativas sanas del $65,00 \%$ (Tabla 11). La reducción en el porcentaje global de aciertos se puede atribuir a que a medida que nos alejamos del momento del fracaso el número de áreas de la empresa que emiten señales de alerta de esta circunstancia es cada vez menor.

\section{CONCLUSIONES}

Las cooperativas son sociedades de marcado carácter social que se rigen por una serie de principios o características que las hacen diferentes de las sociedades mercantiles o capitalistas, entre los que podemos destacar el capital variable y su remuneración, el reparto del resultado en función de las operaciones realizadas por cada socio y la asignación obligatoria de parte de este resultado a la educación, formación y promoción de sus socios y trabajadores.

Estas características diferenciadoras hacen que cuenten con una normativa específica y que normalmente no se hayan considerado en los múltiples estudios que se han hecho sobre previsión del fracaso empresarial. Sin embargo, la nor- 
mativa contable cada vez las acerca más a la hora de confeccionar sus estados contables a los modelos globalizados del resto de sociedades, lo que nos debe permitir ir incluyéndolas en los diferentes estudios y análisis para poder comparar sus resultados con el resto de empresas, aunque poniendo de manifiesto su especificidades.

Se ha trabajado con una muestra compuesta en su totalidad por empresas cooperativas de naturaleza no financiera. En todos los casos la información que se ha procesado se deriva de cuentas anuales en formato abreviado y, por tanto, no sometida a controles externos de auditoría, lo que en principio, podría suponer algunas deficiencias en la calidad y cuestionar su capacidad para ser utilizada en este tipo de análisis. A pesar de ello, el elevado grado de acierto alcanzado nos viene a confirmar que los modelos funcionan y, por consiguiente, que la información aportada por las empresas de este segmento es válida para acometer estas investigaciones y obtener resultados consistentes.

Para la realización del estudio empírico sobre insolvencia empresarial de las cooperativas nos hemos apoyado en un modelo de predicción del fracaso basado en la Regresión Logística Binaria. Como variables independientes se han considerado diferentes ratios económico-financieros de las sociedades cooperativas. Se ha recurrido al análisis factorial de componentes principales y a los resultados de la matriz de correlaciones para seleccionar solamente aquellos ratios que muestren capacidad de añadir globalmente información no redundante y evitar de este modo los problemas y distorsiones en los resultados derivados de la presencia de multicolinealidad. Todo este proceso se ha realizado por separado para los tres años anteriores a la fecha del fracaso de la sociedad cooperativa.

Los resultados de los modelos estimados en el estudio estadístico en los tres años anteriores al fracaso alcanzan porcentajes de acierto aceptables, que van desde el $85 \%$ del año previo al fracaso, al $72,5 \%$ del tercer año. Al igual que sucede en otros trabajos que estudian el fracaso empresarial el porcentaje global de aciertos disminuye a medida que el modelo se aleja en el tiempo de la fecha de fracaso.

El número de variables significativas que incluyen los modelos estimados es mayor el año anterior al fracaso, lo que se traduce en que las áreas desequilibradas de la empresa que emiten señales de alerta son más numerosas a medida que se acerca la fecha del fracaso.

En el primer año anterior al fracaso resulta determinante en el modelo de predicción una variable de solvencia a largo plazo y con menor peso una variable de rentabilidad. Pero lo más significativo es que, en contra de lo que sería lo esperado en cualquier empresa, el coeficiente de esta variable es positivo. Sin embargo, no debemos olvidar que la rentabilidad de una cooperativa medida a través del resultado contable no es comparable con el resto de empresas ya que no tiene en cuenta los posibles beneficios de sus socios vía precios de los pro- 
ductos y servicios cooperativizados. De esta forma, teniendo en cuenta ésta y otras características diferenciadoras de las cooperativas, entendemos que el signo positivo en la variable de rentabilidad podría tener su justificación en que, ante una inminente situación de fracaso de la cooperativa, es muy probable que una gran cantidad de socios soliciten la baja y que para compensar esa pérdida de recursos financieros la cooperativa trate de aumentar la autofinanciación incrementando el resultado contable reduciendo costes, es decir, reduciendo los precios liquidados a sus socios.

En el segundo y tercer año anterior al fracaso tiene una gran relevancia la estructura financiera, de modo que una mayor presencia de gastos financieros o un menor monto de disponible pueden desencadenar la situación concursal de la cooperativa.

Finalmente, nos gustaría conferirle al estudio un carácter de continuidad pues consideramos muy interesante volver a realizar este tipo de análisis a partir de 2011, cuando los datos estén disponibles, ya que después de unos años de transición y tras los referidos cambios, la información ofrecida por las cooperativas será más fiable y comparable, no solo con otras cooperativas sino también con el resto de empresas.

\section{REFERENCIAS BIBLIOGRÁFICAS}

AFONSO RODRÍGUEZ, J. A.; BRUNO PÉREZ, N. A. y GINER RUBIO, J. (2004). "Un análisis univariante y multivariante de la diversificación de carteras bajo heterocedasticidad condicionada". Estudios de Economía Aplicada, vol. 22-2, pp. 1-25.

CRASWELL, A.T. (1999). Does the provision of non-audit servicers impair auditor independence? International Journal of Auditing, 3.

DEMARIS, A. (1992). Logit Modelling: Practical Applications. (Sage Publications, California).

DIÉGUEZ, J.; TRUJILLO, F. y CISNEROS, A.J. (2006): "Modelos de predicción de la insolvencia empresarial: la incorporación de ratios a partir de un marco teórico", VI Jornadas sobre Predicción del Insolvencia Empresarial.

HOSMER, D.W. and LEMESHOW, S. (1989). Applied Logistic Regression. (Wiley: New York).

JUDGE, G.G.; HILL, R.C.; GRIFFITHS, W.E.; LUTKEPOHL, H. and LEE, T.C. (1988). Introduction to the Theory and Practice of Econometrics. (2nd ed., New York, NY: Wiley). 
JUSTE CARRIÓN, J.J.; GÓMEZ GARCÍA, J.M. y FERNÁNDEZ ARUFE, J.E. (2011): "Economía social y desarrollo local/rural. Un análisis de sus sinergias". Estudios de Economía Aplicada, Vol. 29-1, pp. 189-222.

INTRODUCTION TO SAS (2012). UCLA: Academic Technology Services, Statistical Consulting Group. Obtenido de http://www.ats.ucla.edu/stat/stata/ output/stata_logistic.htm (acceso mayo 2012).

ITURRIOZ DEL CAMPO, J. (2010): "Los procesos concursales en situación de crisis: características de sus aplicación a las sociedades cooperativas", $R E$ VESCO: revista de estudios cooperativos, $\mathrm{n}^{\circ} 100$, pp. 134-159.

ITURRIOZ DEL CAMPO, J. y MARTÍN LÓPEZ, S. (2010): "Algunas especialidades financieras del concurso de acreedores de la sociedad cooperativa", Anuario de derecho concursal, $\mathrm{n}^{\circ}$ 19, pp. 189-207.

LABATUT SERER, G.; POZUELO CAMPILLO, J. y VERES FERRER, E. (2009): "Modelización temporal de los ratios contables en la detección del fracaso empresarial de la PYME española", Revista Española de Financiación y Contabilidad, $\mathrm{n}^{\circ} 143$, pp. 423-447.

LEY 5/2011, de 29 de marzo, de Economía Social.

MATEOS RONCO, A.; MARÍN SÁNCHEZ M.M.; MARÍ VIDAL, S. y SEGUÍ MAS, E. (2011): "Los modelos de predicción del fracaso empresarial y su aplicabilidad en cooperativas agrarias", CIRIEC-España, Revista de Economía Pública, Social y Cooperativa, $n^{\circ} 70$, abril, pp. 179-208.

ORDEN ECO/3614/2003, de 16 de diciembre, por la que se aprueban las normas sobre los aspectos contables de las Sociedades Cooperativas.

ORDEN EHA/3360/2010, de 21 de diciembre, por la que se aprueban las normas sobre los aspectos contables de las sociedades cooperativas.

POZUELO CAMPILLO, J.; LABATUT SERER, G. y VERES FERRER, E. (2010): "Análisis descriptivo de los procesos de fracaso empresarial en microempresas mediante técnicas multivariantes", Revista Europea de Dirección y Economía de la Empresa, vol. 19, n 3, pp. 47-66.

RODRÍGUEZ HERNÁNDEZ, J.E. y BARRIOS GARCÍA, J.A. (2003). "Un modelo logit mixto de tenencia de vivienda en Canarias". Estudios de Economía Aplicada, vol. 21-I. pp. 173-191.

SHARMA, D.S. and SIDHU, J. (2001). Professionalism vs. Commercialism: the association between mon-audit services (NAS) and audit independence. Journal of Business Finance and Accounting, 28.

VEALL, M.R. and ZIMMERMANN, K.F (1994). Goodness of Fit measures in the Tobit model. Oxford Bulletin of Economics and Statistics, 56.

VERES FERRER, E.; LABATUT SERER, G. y POZUELO CAMPILLO, J. (2009): "Hacia una ordenación de las pequeñas empresas atendiendo a su posible situación e fracaso", Estudios de Economía Aplicada, vol. 27-3, pp. $1-18$. 
